\title{
Antimicrobial activity of ceftobiprole against Gram-negative and Gram-positive pathogens: results from INVITA-A-CEFTO Brazilian study
}

\begin{abstract}
Ceftobiprole is a broad-spectrum cephalosporin with potent activity against staphylococci, including those resistant to oxacillin, as well as against most Gram-negative bacilli including Pseudomonas aeruginosa. In this study, the in vitro activity of ceftobiprole and comparator agents was tested against bacterial isolates recently collected from Brazilian private hospitals. A total of 336 unique bacterial isolates were collected from hospitalized patients between February 2008 and August 2009. Each hospital was asked to submit 100 single bacterial isolates responsible for causing blood, lower respiratory tract or skin and soft tissue infections. Bacterial identification was confirmed and antimicrobial susceptibility testing was performed using CLSI microdilution method at a central laboratory. The CLSI M100-S21 (2011) was used for interpretation of the antimicrobial susceptibility results. Among the 336 pathogens collected, 255 (75.9\%) were Gram-negative bacilli and 81 (24.1\%) were Gram-positive cocci. Although ceftobiprole $\mathrm{MIC}_{50}$ values for oxacillin resistant strains were two-fold higher than for methicillin susceptible S. aureus, ceftobiprole inhibited $100 \%$ of tested S. aureus at MICs $\leq 4 \mu \mathrm{g} / \mathrm{mL}$. Polymyxin B was the only agent to show potent activity against Acinetobacter spp. $\left(\mathrm{MIC}_{50 / 9}, 0.5 / 1 \mu \mathrm{g} / \mathrm{mL}\right.$ ), and P. aeruginosa $\left(\mathrm{MIC}_{50 / 90}, 1 / 2 \mu \mathrm{g} / \mathrm{mL}\right)$. Resistance to broadspectrum cephalosporins varied from $55.3-68.5 \%$ and $14.3-28.5 \%$ among E. coli and Klebsiella spp. isolates, respectively; with ceftobiprole $\mathrm{MIC}_{50}>6 \mu \mathrm{g} / \mathrm{mL}$ for both species. Our results showed that ceftobiprole has potent activity against staphylococci and E. faecalis, which was superior to that of vancomycin. Our data also indicates that ceftobiprole demonstrated potency comparable to that of cefepime and ceftazidime against key Gram-negative species.
\end{abstract}

Keywords: cephalosporins; Brazil; Gram-negative aerobic bacteria; methicillin-resistant Staphylococcus aureus.

\section{INTRODUCTION}

Fourth-generation cephalosporins represent a valuable addition to the therapeutic armamentarium since they have demonstrated activity against Gram-negative bacilli, including Enterobacteriaceae and, as well as Gram-positive cocci such as Streptococcus pneumoniae and methicllin-suceptible staphylococci. However, the lack of activity against methicillin-resistant staphylococci and extended-spectrum beta-lactamases (ESBL)-producing isolates has limited wider clinical use. ${ }^{1,2}$ According to the most recent SENTRY data report, Staphylococcus aureus $(20.2 \%)$ and coagulase-negative staphylococci (CoNS; 14.5\%) ranked as the first and second cause of bloodstream infections in
Brazilian hospitals between 2005 and 2008. ${ }^{3}$ $S$. aureus was also the most common cause of skin and soft tissue infections (28.1\%) and was isolated from $24.9 \%$ of patients with pneumonia. In that study, $31.0 \%$ and $78.7 \%$ of $S$. aureus and CoNS showed resistance to methicillin. The limited number of approved drugs with activity against multidrug-resistant bacteria such as methicillin-resistant S. aureus (MRSA) has increased the demand for new agents with a novel mechanism of action or an ability to overcome bacterial resistance.

Ceftobiprole is a pyrrolidinone-3-ylidenemethyl cephalosporin with a broad-spectrum of activity against Gram-positive cocci and Gram-negative bacilli. ${ }^{4-6}$ The binding of ceftobiprole to penicillin-binding proteins
Authors

Rosângela Ferraz Cereda ${ }^{1}$ Heber Dias Azevedo ${ }^{2}$ Raquel Girardello 3

Danilo Elias Xavier Ana C Gales ${ }^{5}$

INVITA-A-CEFTO

Brazilian Study Group ${ }^{6}$

${ }^{1} \mathrm{PhD}$ in Medicine; Latin America Medical Manager at Janssen-Cilag, Brazil ${ }^{2} \mathrm{MD}$, Infectology; Medical Manager at Janssen-Cilag, Brazil

${ }^{3}$ Biologist; PhD Student at the Post-graduation Course in Sciences, Universidade

Federal de São Paulo (UNIFESP), Brazil

${ }^{4}$ Pharmacist, PhD Student at the Post-graduation Course in Sciences, UNIFESP, Brazil ${ }^{5} \mathrm{PhD}$ in Medicine; Professor of Infectology, UNIFESP, Brazil

${ }^{6}$ Marinês D V Martino (Hospital Israelita Albert Einstein, São Paulo, SP); Ronaldo Rosembaum (Hospital Samaritano, Rio de Janeiro, RJ); Silvana B Ricardo (Hospital Matter Dei, Belo Horizonte, MG); Rosângela

C Souza (Hospital São

Domingos, São Luis, MA)

Submitted on: 01/13/2011 Approved on: 03/09/2011

Correspondence to: Ana Gales

Rua Leandro Dupret, 188 04025-010, São Paulo, SP Brazil

ana.gales@gmail.com

Financial Support: This study was supported by an educational/research grant from Janssen-Cilag.

Conflict of interest: ACG has received research funding and/ or consultation fees from Janssen-Cilag, Pfizer,

Novartis, and SanofiAventis. Other authors have nothing to declare.

(C)2011 Elsevier Editora Ltda. All rights reserved. 
(PBPs) is a critical determinant of its antibacterial activity. ${ }^{4}$ Ceftobiprole demonstrated potent binding to PBPs from Gram-positive bacteria, including those with decreased $\beta$-lactam sensitivity, such as PBP2a in MRSA and PBP2x in penicillin-resistant $S$. pneumoniae (PRSP), in contrast to ceftriaxone. In Escherichia coli, ceftobiprole exhibited strong binding to the essential PBPs, PBP2 and PBP3. It also exhibited a binding profile similar to those of cefepime and ceftazidime in $P$. aeruginosa but with enhanced binding to PBP2. These binding profiles explain the broad-spectrum activity for ceftobiprole. ${ }^{5}$ In addition, in single-step and serial passage in vitro resistance development studies, ceftobiprole demonstrated a low propensity to select for resistant subpopulations. ${ }^{7,8}$ The purpose of this study was to assess the in vitro activity of ceftobiprole and comparator agents against Gram-positive cocci and Gram-negative bacilli, recently isolated from patients of Brazilian private hospitals.

\section{MATERIAL AND METHODS}

\section{Participant medical centers}

Four private hospitals participated of the INVITA-A-CEFTO Brazilian Study. The medical centers were located in four distinct Brazilian cities, Belo Horizonte, São Paulo, Rio de Janeiro and São Luís. Selection of the participant medical centers was based on the criteria that they should have preferentially $\geq 200$ beds, at least one adult intensive care unit and located in cities with more than one million inhabitants.

\section{Bacterial isolates}

A total of 336 consecutive isolates of Gram-positive cocci and Gram-negative bacilli was submitted between February 2008 and August 2009. Each medical center was guided by protocol to submit Gram-positive cocci and Gramnegative bacilli collected from patients with diagnosis of pneumonia (40 isolates being from 20 ventilator-assisted patients), bloodstream infections (40 isolates) and skin and soft-tissue infections (20 isolates), according to the Centers for Disease Control and Prevention (CDC) definitions. ${ }^{9}$ One isolate per patient was evaluated. All isolates were identified at the participating institution by routine methodologies in use at each laboratory. Upon receipt at the central laboratory (UNIFESP, São Paulo), isolates were subcultured to ensure viability and purity. Confirmation of species identification was performed with the BD Phoenix $^{\mathrm{Tm}}$ Automated Microbiology System (BD Diagnostics, $\mathrm{MD}$, USA) or conventional methods, as required.

\section{Susceptibility testing}

Antimicrobial susceptibility testing was performed by the broth microdilution method, following recommendations of the Clinical and Laboratory Standards Institute (CLSI). ${ }^{10}$ Antimicrobial powders were obtained from the respective manufacturers and microdilution plates were prepared by TREK Diagnostics (West Sussex, England). Susceptibility results were interpreted according to CLSI document M100-S2 $1^{11}$ for all comparison agents except for doripenem, ${ }^{12}$ tigecycline ${ }^{13}$ and ceftobiprole. ${ }^{14}$ Quality control was performed by testing E. coli ATCC 25922; P. aeruginosa ATCC 27853; S. aureus ATCC 29213 and E. faecalis ATCC 29212.

\section{RESULTS}

A total of 336 isolates were collected as part of the INVITA-A-CEFTO Brazilian Study between February 2008 and August 2009. Of those, 225 (75.9\%) and 81 (24.1\%) were classified as Gram-negative and Gram-positive, respectively. The bacterial isolates were collected mainly from patients diagnosed with bloodstream (38.4\%), lower respiratory tract (39.9\%) and skin and soft tissue (21.7\%) infections. The frequency of isolates collected, according to infection type and medical centers is shown in Table 1 . The most frequent genera/species collected were

Table 1. Frequencies of isolates collected by the INVITA-A-CEFTO study according to type of infection and medical center

\begin{tabular}{|lcccc|}
\hline \multicolumn{1}{c}{$\begin{array}{c}\text { Medical center } \\
\text { (city/state) }\end{array}$} & $\begin{array}{c}\text { Bloodstream } \\
\text { infections }\end{array}$ & $\begin{array}{c}\text { Respiratory tracts } \\
\text { infections }\end{array}$ & $\begin{array}{c}\text { Skin and soft tissue } \\
\text { infection }\end{array}$ & Total \\
\hline 01 (São Paulo, SP) & 40 & 34 & 20 & $94(28.0 \%)$ \\
\hline 02 (Rio de Janeiro, RJ) & 38 & 39 & 20 & $97(28.9 \%)$ \\
\hline 03 (Belo Horizonte, MG) & 24 & 21 & 15 & $60(17.9 \%)$ \\
\hline 04 (São Luis, MA) & 27 & 40 & 18 & $85(25.3 \%)$ \\
\hline Total & $129(38.4 \%)$ & $134(39.9 \%)$ & $73(21.7 \%)$ & $336(100 \%)$ \\
\hline
\end{tabular}


Table 2. Frequency of isolates collected by the INVITA-A-CEFTO Brazilian Study

\begin{tabular}{|c|c|}
\hline Microorganism & Number of isolates (\%) \\
\hline Pseudomonas spp. ${ }^{\mathrm{a}}$ & $96(28.6)$ \\
\hline Klebsiella spp. ${ }^{\mathrm{b}}$ & $41(12.2)$ \\
\hline Staphylococcus aureus & $36(10.7)$ \\
\hline Acinetobacter baumannii & $30(8.9)$ \\
\hline Escherichia coli & $28(8.3)$ \\
\hline Staphylococcus coagulase negativa ${ }^{c}$ & $23(6.8)$ \\
\hline Enterobacter spp. ${ }^{\mathrm{d}}$ & $21(6.3)$ \\
\hline Enterococcus faecalis & $18(5.4)$ \\
\hline Serratia spp..$^{\mathrm{e}}$ & $17(5.1)$ \\
\hline Morganella morgannii & $4(1.2)$ \\
\hline Proteus mirabilis & $4(1.2)$ \\
\hline Providencia stuartii & $4(1.2)$ \\
\hline Streptococcus spp. ${ }^{\mathrm{f}}$ & $4(1.2)$ \\
\hline Stenotrophomonas maltophilia & $3(0.9)$ \\
\hline Burkholderia cерасеа & $2(0.6)$ \\
\hline Achromobacter xylosoxidans & $1(0.3)$ \\
\hline Aeromonas hydrophila & $1(0.3)$ \\
\hline Citrobacter koseri & $1(0.3)$ \\
\hline Cryseobacterium gleum & $1(0.3)$ \\
\hline Moraxella spp. & $1(0.3)$ \\
\hline Total & 336 \\
\hline
\end{tabular}

a P. aeruginosa (94), P. putida (1), P. fluorescens (1).

${ }^{\mathrm{b}}$ K. pneumoniae (38), K. oxytoca (3).

' $S$. epidermidis (15), S. haemolyticus (2), S. hominis (2),

S. capitis (1), Staphylococcus spp. (3).

${ }^{\mathrm{d}}$ E. cloacae (10), E. aerogenes (9), E. cancerogenus (1),

E. sakazakii (1). Recentely, E. sakazakii was called Cronobac-

ter sakazakii.

eS. marcescens (14), S. plymuthica (3).

${ }^{\mathrm{f}} S$. mitis (1), S. agalactiae (3).

Pseudomonas spp. (28.6\%); Klebsiella spp. (12.2\%); S. aureus (10.7\%); Acinetobacter spp. (8.9\%); E. coli $(8.3 \%)$ and coagulase negative staphylococci (CoNS, $6.8 \%)$. The frequency of occurrence of all pathogens collected is shown in Table 2.

Antimicrobial activity of the tested agents and the susceptibility profile of the most frequent Gram-negative isolates are shown in Table 3. Ceftobiprole showed similar activity to that displayed by cefepime against $P$. aeruginosa, K. pneumoniae, A. baumannii, E. coli and Enterobacter spp.

$P$. aeruginosa showed the highest rates of susceptibility towards polymyxin B (98.9\%), amikacin (59.1\%) and piperacillin/tazobactam (58.1\%) (Table 3). Among the cephalosporins, slightly greater percentage of isolates was inhibited at MICs $\leq 8 \mu \mathrm{g} / \mathrm{mL}$ by ceftazidime $(48.4 \%)$ or cefepime ( $47.8 \%)$ than by ceftobiprole ( $36.8 \%)$. Ceftobiprole and ceftriaxone inhibited $36.8 \%$ of K. pneumoniae isolates at concentrations $\leq 8 \mu \mathrm{g} / \mathrm{mL}$. Against this species, ceftobiprole activity was comparable to that displayed by cefepime (39.5\%), but lower than that of ceftazidime (44.7\%, Table 4). Among the carbapenems, meropenem (76.3\% susceptibility) and doripenem (73.7\% susceptibility) showed remarkable susceptibility rates, followed by imipenem (68.4\% susceptibility). Moreover, imipenem $\left(\mathrm{MIC}_{90}, 4 \mu \mathrm{g} / \mathrm{mL}\right)$ was two-fold more potent than meropenem $\left(\mathrm{MIC}_{90}, 8 \mu \mathrm{g} / \mathrm{mL}\right)$ and doripenem $\left(\mathrm{MIC}_{90}, 8 \mu \mathrm{g} / \mathrm{mL}\right)$ against $K$. pneumoniae isolates. All tested K. pneumoniae isolates were susceptible to tigecycline. Levofloxacin resistance was observed in $47.4 \%$ of K. pneumoniae isolates.

All tested A. baumannii isolates were susceptible to tigecycline $\left(\mathrm{MIC}_{90}, 0.5 \mu \mathrm{g} / \mathrm{mL}\right)$ and polymyxin $\mathrm{B}\left(\mathrm{MIC}_{90}\right.$, $\leq 0.5 \mu \mathrm{g} / \mathrm{mL}$ ). Ceftobiprole had $\mathrm{MIC}_{50 / 90},>6 \mu \mathrm{g} / \mathrm{mL}$ as did cefepime and ceftazidime.

The potency of ceftobiprole $\left(\mathrm{MIC}_{50}, 0.5 \mu \mathrm{g} / \mathrm{mL}\right.$ ) wasidentical to those displayed by ceftazidime $\left(\mathrm{MIC}_{50}, 0.5 \mu \mathrm{g} / \mathrm{mL}\right)$ and cefepime $\left(\mathrm{MIC}_{50}, 0.5 \mu \mathrm{g} / \mathrm{mL}\right)$ among E. coli isolates. Against Enterobacter spp., cefepime ( $\mathrm{MIC}_{50}, \leq 0.12 \mu \mathrm{g} / \mathrm{mL}$ ) was at least two-fold and four-fold more active than ceftobiprole $\left(\mathrm{MIC}_{50}, 0.25 \mu \mathrm{g} / \mathrm{mL}\right)$ and ceftazidime $\left(\mathrm{MIC}_{50}\right.$, $0.5 \mu \mathrm{g} / \mathrm{mL}$ ), respectively. Nevertheless, the highest in vitro activity for ceftobiprole was observed for this genus. Approximately $68 \%$ and $76 \%$ of E. coli and Enterobacter spp., respectively, were inhibited by ceftobiprole at $8 \mu \mathrm{g} / \mathrm{mL}$ (Table 4).

The activity of ceftobiprole and other antimicrobial agents tested against Gram-positive isolates is shown in Tables 5 and 6 . Overall, $33.3 \%$ of $S$. aureus isolates were resistant to oxacillin. Ceftobiprole was two-fold more potent against oxacillin-susceptible $S$. aureus $\left(\mathrm{MIC}_{50}, 0.5 \mu \mathrm{g} / \mathrm{mL}\right)$ than oxacillin-resistant $S$. aureus $\left(\mathrm{MIC}_{50}, 1 \mu \mathrm{g} / \mathrm{mL}\right)$. All S. aureus strains were inhibited by concentrations of ceftobiprole $\leq 4 \mu \mathrm{g} / \mathrm{mL}$. Agents providing the highest coverage against all S. aureus included vancomycin $\left(\mathrm{MIC}_{50}, 1 \mu \mathrm{g} / \mathrm{mL}\right.$; $100 \%$ susceptible), teicoplanin $\left(\mathrm{MIC}_{50}, \leq 2 \mu \mathrm{g} / \mathrm{mL} ; 100 \%\right.$ susceptible), linezolid ( $\mathrm{MIC}_{50}, 4 \mu \mathrm{g} / \mathrm{mL} ; 97.2 \%$ susceptible) and tigecycline $\left(\mathrm{MIC}_{50}, 0.25 \mu \mathrm{g} / \mathrm{mL} ; 97.2 \%\right.$ susceptible). Tigecycline $\left(\mathrm{MIC}_{50}, 0.25 \mu \mathrm{g} / \mathrm{mL} ; 100 \%\right.$ susceptibility) followed by ceftobiprole $\left(\mathrm{MIC}_{50 / 90}, 1\right.$ and $8 \mu \mathrm{g} / \mathrm{mL}$ ) were the most active agents tested against CoNS isolates. At $2 \mu \mathrm{g} / \mathrm{mL}$, ceftobiprole inhibited $95.7 \%$ of CoNS isolates (Table 6). Only a single CoNS isolate showed a ceftobiprole MIC of $8 \mu \mathrm{g} / \mathrm{mL}$.

Ceftobiprole $\left(\mathrm{MIC}_{50 / 9}, 0.5\right.$ and $16 \mu \mathrm{g} / \mathrm{mL}$ ) inhibited the growth of $88.9 \%$ of the E. faecalis isolates at concentrations 
Table 3. In vitro activity of ceftobiprole in comparison to selected antimicrobial agents tested against the main Gram negative pathogens collected by the INVITA-A-CEFTO Brazilian Study

\begin{tabular}{|c|c|c|c|c|}
\hline \multirow{2}{*}{$\begin{array}{l}\text { Organism/ } \\
\text { Antimicrobial agents }^{\mathrm{a}}\end{array}$} & \multicolumn{2}{|c|}{ MIC $(\mu \mathrm{g} / \mathrm{mL})$} & \multirow[b]{2}{*}{ Susceptible (\%) } & \multirow[b]{2}{*}{ Resistant (\%) } \\
\hline & $\mathrm{MIC}_{50}$ & $\mathrm{MIC}_{90}$ & & \\
\hline \multicolumn{5}{|l|}{ P. aeruginosa (94) } \\
\hline Ceftobiprole & $>16$ & $>16$ & $15.1^{\mathrm{c}}$ & $84.9^{c}$ \\
\hline Ceftazidime & 16 & $>16$ & 48.4 & 45.2 \\
\hline Cefepime & 16 & $>16$ & 47.3 & 43.0 \\
\hline Aztreonam & 16 & $>16$ & 32.3 & 50.5 \\
\hline Piperaracillin/Tazobactam & 64 & $>64$ & 58.1 & 41.9 \\
\hline Doripenem $^{b}$ & 4 & $>16$ & 40.9 & $-f$ \\
\hline Meropenem & 8 & $>8$ & 43.6 & 46.8 \\
\hline Imipenem & 8 & $>8$ & 43.0 & 44.1 \\
\hline Ciprofloxacin & $>2$ & $>2$ & 37.6 & 59.1 \\
\hline Levofloxacin & $>4$ & $>4$ & 34.4 & 59.1 \\
\hline Amikacin & 16 & $>32$ & 59.1 & 37.6 \\
\hline Polymyxin B & 1 & 2 & 98.9 & 1.1 \\
\hline \multicolumn{5}{|l|}{ K. pneumoniae (38) } \\
\hline Ceftobiprole & $>16$ & $>16$ & $31.6^{\mathrm{c}}$ & $68.5^{\mathrm{c}}$ \\
\hline Cefoxitin & 8 & $>16$ & 60.5 & 31.6 \\
\hline Ceftriaxone & $>32$ & $>32$ & 28.9 & 68.5 \\
\hline Ceftazidime & $>16$ & $>16$ & 42.1 & 55.3 \\
\hline Cefepime & $>16$ & $>16$ & 39.5 & 57.9 \\
\hline Aztreonam & $>16$ & $>16$ & 42.1 & 55.3 \\
\hline Piperaracillin/Tazobactam & 64 & $>64$ & 39.5 & 52.6 \\
\hline Doripenem $^{\mathrm{b}}$ & 0.25 & 8 & 73.7 & 23.6 \\
\hline Meropenem & 0.12 & 8 & 76.3 & 21.1 \\
\hline Imipenem & 1 & 4 & 68.4 & 13.2 \\
\hline Ciprofloxacin & $>2$ & $>2$ & 34.2 & 63.2 \\
\hline Levofloxacin & 4 & $>4$ & 39.5 & 47.4 \\
\hline Amikacin & 8 & 32 & 84.2 & 2.6 \\
\hline Polymyxin $B^{e}$ & $\leq 0.5$ & 1 & 100.0 & 0.0 \\
\hline Tigecycline $^{\mathrm{b}}$ & 1 & 2 & 100.0 & 0.0 \\
\hline \multicolumn{5}{|l|}{ A. baumannii (30) } \\
\hline Ceftobiprole & $>16$ & $>16$ & $20.0^{c}$ & $80.0^{c}$ \\
\hline Ceftriaxone & $>32$ & $>32$ & 6.7 & 66.7 \\
\hline Ceftazidime & $>16$ & $>16$ & 40.0 & 56.7 \\
\hline Cefepime & $>16$ & $>16$ & 26.7 & 73.3 \\
\hline Piperaracillin/Tazobactam & $>64$ & $>64$ & 16.7 & 76.7 \\
\hline Doripenem $^{\mathrm{b}}$ & $>16$ & $>16$ & 23.3 & $-f$ \\
\hline Meropenem & $>8$ & $>8$ & 23.3 & 76.7 \\
\hline Imipenem & $>8$ & $>8$ & 23.3 & 76.7 \\
\hline Ciprofloxacin & $>2$ & $>2$ & 20.0 & 80.0 \\
\hline Levofloxacin & $>4$ & $>4$ & 20.0 & 76.7 \\
\hline Amikacin & $>32$ & $>32$ & 43.3 & 56.7 \\
\hline Polymyxin B & $\leq 0.5$ & 1 & 100.0 & 0.0 \\
\hline Tigecycline $^{\mathrm{b}}$ & 0.5 & 2 & 100.0 & 0.0 \\
\hline
\end{tabular}


Table 3. In vitro activity of ceftobiprole in comparison to selected antimicrobial agents tested against the main Gram negative pathogens collected by the INVITA-A-CEFTO Brazilian Study

\begin{tabular}{|c|c|c|c|c|}
\hline \multirow{2}{*}{$\begin{array}{l}\text { Organism/ } \\
\text { Antimicrobial agents }^{\mathrm{a}}\end{array}$} & \multicolumn{2}{|c|}{ MIC ( $\mu \mathrm{g} / \mathrm{mL})$} & \multirow[b]{2}{*}{ Susceptible (\%) } & \multirow[b]{2}{*}{ Resistant (\%) } \\
\hline & $\mathrm{MIC}_{50}$ & $\mathrm{MIC}_{90}$ & & \\
\hline \multicolumn{5}{|l|}{ E. coli $(28)$} \\
\hline Ceftobiprole & 0.5 & $>16$ & $57.1^{\mathrm{c}}$ & $39.2^{c}$ \\
\hline Cefoxitin & $\leq 4$ & $>16$ & 82.1 & 14.3 \\
\hline Ceftriaxone & 1 & $>32$ & 64.3 & 28.5 \\
\hline Ceftazidime & 0.5 & 16 & $78.6^{\mathrm{d}}$ & 17.8 \\
\hline Cefepime & 0.5 & $>16$ & 78.6 & 14.3 \\
\hline Aztreonam & $\leq 1$ & $>16$ & 75.0 & 14.3 \\
\hline Piperaracillin/Tazobactam & 4 & 16 & 92.9 & 7.1 \\
\hline Doripenem $^{\text {b }}$ & $\leq 0.12$ & 1 & 96.4 & 3.57 \\
\hline Meropenem & $\leq 0.12$ & 0.25 & 100.0 & 0.0 \\
\hline Imipenem & 0.25 & 2 & 89.3 & 0.0 \\
\hline Ciprofloxacin & 2 & $>2$ & 46.4 & 50.0 \\
\hline Levofloxacin & 2 & $>4$ & 50.0 & 46.4 \\
\hline Amikacin & $\leq 4$ & $>32$ & 85.7 & 10.7 \\
\hline Polymyxin Be & $\leq 0.25$ & 1 & 100.0 & 0.0 \\
\hline Tigecycline $^{\mathrm{b}}$ & 0.25 & 1 & 100.0 & 0.0 \\
\hline \multicolumn{5}{|l|}{ Enterobacter spp. (21) } \\
\hline Ceftobiprole & 0.25 & $>16$ & $76.2^{\mathrm{c}}$ & $23.8^{c}$ \\
\hline Ceftriaxone & 1 & $>32$ & 71.4 & 28.6 \\
\hline Ceftazidime & 0.5 & $>16$ & 76.2 & 23.8 \\
\hline Cefepime & $\leq 0.12$ & $>16$ & 76.2 & 23.8 \\
\hline Aztreonam & $\leq 1$ & $>16$ & 66.7 & 28.6 \\
\hline Piperaracillin/Tazobactam & 4 & $>64$ & 76.2 & 19.0 \\
\hline Doripenem $^{\mathrm{b}}$ & 0.5 & 2 & 85.7 & 9.52 \\
\hline Meropenem & 0.12 & 1 & 90.5 & 0.0 \\
\hline Imipenem & 4 & 4 & 28.6 & 57.2 \\
\hline Ciprofloxacin & $\leq 0.25$ & $>2$ & 81.0 & 19.0 \\
\hline Levofloxacin & $\leq 0.5$ & $>4$ & 81.0 & 19.0 \\
\hline Amikacin & $\leq 4$ & 16 & 90.5 & 9.5 \\
\hline Polymyxin Be & 1 & $>4$ & 71.4 & 23.8 \\
\hline Tigecycline $^{b}$ & 0.5 & 2 & 95.2 & 0.0 \\
\hline
\end{tabular}

${ }^{a}$ MIC determined according CLSI (2009) recommendations.

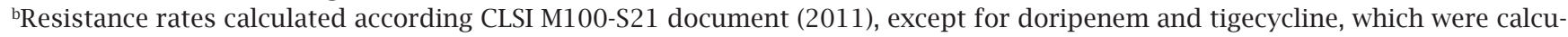
lated according the FDA criteria.

'Interpretative criteria according to Rossolini et al. ${ }^{14}$

${ }^{\mathrm{d}}$ According to breakpoints established by CLSI for P. aeruginosa $(\leq 2 \mu \mathrm{g} / \mathrm{mL}$ for susceptibility and $\geq 8 \mu \mathrm{g} / \mathrm{mL}$ for resistance).

eAccording P. aeruginosa breakpoint, CLSI 2009 recommendations.

fInterpretative criteria not established by CLSI or FDA. 
Table 4. Comparisons of cephalosporins tested against the major Gram-negative isolates collected by the INVITA-A-CEFTO Brazilian Study

\begin{tabular}{|c|c|c|c|c|c|c|c|c|c|c|}
\hline \multirow[t]{2}{*}{ Organism (n) } & \multirow{2}{*}{$\begin{array}{l}\text { Antimicrobial } \\
\text { agents }\end{array}$} & \multicolumn{9}{|c|}{ Cumulative $\%$ inhibited at MIC $(\mu \mathrm{g} / \mathrm{mL})$} \\
\hline & & 0.12 & 0.25 & 0.5 & 1 & 2 & 4 & 8 & 16 & 32 \\
\hline \multirow[t]{3}{*}{ P. aeruginosa (94) } & Ceftobiprole & & & 1.1 & 2.1 & 6.3 & 14.7 & 36.8 & 45.3 & 100.0 \\
\hline & Cefepime & & & & & 5.3 & 30.5 & 47.8 & 56.8 & 100.0 \\
\hline & Ceftazidime & & & & 2.1 & 7.4 & 35.8 & 48.4 & 54.7 & 100.0 \\
\hline \multirow[t]{4}{*}{ K. pneumoniae (38) } & Ceftobiprole & 21.1 & 28.9 & & 31.6 & & & 36.8 & & 100.0 \\
\hline & Cefepime & & 28.9 & 34.2 & & 36.8 & & 39.5 & 42.1 & 100.0 \\
\hline & Ceftazidime & & 21.1 & 28.9 & 34.2 & 39.5 & 42.1 & 44.7 & 100.0 & \\
\hline & Ceftriaxone & 26.3 & & 28.9 & & 31.6 & & 36.8 & 100.0 & \\
\hline \multirow[t]{4}{*}{ A. baumannii (30) } & Ceftobiprole & & 10.0 & 16.7 & & 20.0 & & & 30.0 & 100.0 \\
\hline & Cefepime & & 3.3 & & 10.0 & 20.0 & 23.3 & 26.7 & & 100.0 \\
\hline & Ceftazidime & & & & 3.3 & 6.7 & 26.7 & 40.0 & 43.3 & 100.0 \\
\hline & Ceftriaxone & & & & & 3.3 & & 6.7 & 33.3 & 40.0 \\
\hline \multirow[t]{4}{*}{ E. coli (28) } & Ceftobiprole & & 7.1 & 50.0 & 57.1 & & 60.7 & 67.9 & & 100.0 \\
\hline & Cefepime & & 46.4 & 50.0 & & 64.3 & 71.4 & 78.6 & 85.7 & 100.0 \\
\hline & Ceftazidime & & 39.3 & 57.1 & 64.3 & 71.4 & 78.6 & 82.1 & 92.9 & 100.0 \\
\hline & Ceftriaxone & 7.1 & 60.7 & 89.3 & 92.9 & 100.0 & & & & \\
\hline \multirow[t]{4}{*}{ Enterobacter spp. (21) } & Ceftobiprole & & 66.7 & 76.2 & & & & & & 100.0 \\
\hline & Cefepime & & 61.9 & 71.4 & & & & 76.2 & & 100.0 \\
\hline & Ceftazidime & & 38.1 & 71.4 & 76.2 & & & & & 100.0 \\
\hline & Ceftriaxone & & 47.6 & & 71.4 & & 76.2 & & & 100.0 \\
\hline
\end{tabular}

of $1 \mu \mathrm{g} / \mathrm{mL}$ even though enterococci are generally not inhibited by cephalosporins. Only two (20.2\%) E. faecalis strains isolated from a single medical center presented ceftobiprole MICs $\geq 16 \mu \mathrm{g} / \mathrm{mL}$ (data not shown). Among the E. faecalis isolates tested, $94.4 \%$ were susceptible to ampicillin, vancomycin and teicoplanin, representing the highest susceptible rates (Table 5). One linezolid-resistant E. faecalis isolate (5.6\%) was isolated from a patient diagnosed with skin and soft tissue infection.

\section{DISCUSSION}

The aim of this study was to assess the activity of ceftobiprole, a new broad-spectrum cephalosporin, against contemporary Gram-positive and Gram-negative pathogens collected from patients hospitalized at four distinct Brazilian medical centers, as part of the INVITA-A-CEFTO Brazilian Study.

Ceftobiprole was highly potent against all staphylococcal isolates, inhibiting $100 \%$ of CoNS and S. aureus at MICs $\leq 8 \mu \mathrm{g} / \mathrm{mL}$ and $\leq 4 \mu \mathrm{g} / \mathrm{mL}$, respectively. MIC $_{90}$ values for MRSA were eight-fold higher than those for MSSA isolates (Table 5). These results were similar to those previously reported. ${ }^{7,15,16}$ Although cephalosporins are generally inactive against E. faecalis, ceftobiprole inhibited $88.9 \%$ at MICs $\leq 4 \mu \mathrm{g} / \mathrm{mL}$.
Ceftobiprole showed similar potency to those of 3 th- and 4 th-generation cephems $\left(\mathrm{MIC}_{50}\right.$ values, $0.25 \geq 16 \mu \mathrm{g} / \mathrm{mL}$ ) for the main Enterobacteriaceae species. All cephaloporins demonstrated poor activity against K. pneumoniae (28.9-42.1\% susceptible). This fact could be explained by the probable high rate of ESBL-producing K. pneumoniae isolates in Brazilian hospitals and since ceftobiprole is hydrolyzed by class $\mathrm{A}, \mathrm{B}$, and $\mathrm{D}$ extended-spectrum beta-lactamases, but not by class $\mathrm{C}$ enzymes, as previously reported. ${ }^{17}$ Ceftobiprole, like cefepime, is a weak inducer and a poor substrate for AmpC $\beta$-lactamases. ${ }^{1,17}$

Ceftobiprole like ceftazidime and cefepime $\left(\mathrm{MIC}_{50 / 90} \geq 16 \mu \mathrm{g} / \mathrm{mL}\right.$, Table 3 ) showed poor in vitro activity against $P$. aeruginosa and Acinetobacter spp. isolates. Polymyxin was the only antimicrobial agent to show good activity against both P. aeruginosa and Acinetobacter spp. isolates. The elevated carbapenem resistance rates noticed among $P$. aeruginosa and Acinetobacter spp. tested in this study could be possibly attributed to the spread of clones that produce SPM-1 and OXA-23, respectively, in Brazilian hospitals, as noticed before. ${ }^{18,19}$

Despite the low number of isolates collected, the resistance rates to oxacillin among staphylococci appeared to be similar to those previously reported in a Brazilian study. ${ }^{3}$ Curiously, the antimicrobial resistance rates 
Table 5. In vitro activity of ceftobiprole in comparison to selected antimicrobial agents tested against the main Gram-positive pathogens collected by the INVITA-A-CEFTO Brazilian Study

\begin{tabular}{|c|c|c|c|c|}
\hline \multirow[t]{2}{*}{ Organism/ Antimicrobial agent } & \multicolumn{2}{|c|}{ MIC $(\mu \mathrm{g} / \mathrm{mL})^{\mathrm{a}}$} & \multirow[b]{2}{*}{ Susceptible (\%) } & \multirow[b]{2}{*}{ Resistant (\%) } \\
\hline & $\mathrm{MIC}_{50}$ & $\mathrm{MIC}_{90}$ & & \\
\hline \multicolumn{5}{|l|}{ Enterococcus faecalis (18) } \\
\hline Ceftobiprole & 0.5 & 16 & $88.9^{\mathrm{d}}$ & $11.1^{\mathrm{d}}$ \\
\hline Penicillin & 4 & $>8$ & 88.9 & 11.1 \\
\hline Ampicillin & 1 & 2 & 94.4 & 5.6 \\
\hline Erythromycin & $>4$ & $>4$ & 5.6 & 61.1 \\
\hline Gentamicin & $>8$ & $>8$ & $66.7^{\mathrm{c}}$ & $33.3^{c}$ \\
\hline Linezolid & 2 & $>4$ & 83.3 & 5.6 \\
\hline Levofloxacin & 4 & $>4$ & 44.4 & 44.4 \\
\hline Tigecycline & 0.25 & 0.5 & 66.7 & -e \\
\hline Vancomycin & 2 & 4 & 94.4 & 5.6 \\
\hline Teicoplanin & $\leq 2$ & $\leq 2$ & 94.4 & 5.6 \\
\hline \multicolumn{5}{|l|}{ CoNS (23) } \\
\hline Ceftobiprole & 1 & 8 & $95.7^{\mathrm{d}}$ & $4.3^{\mathrm{d}}$ \\
\hline Penicillin & $>8$ & $>8$ & 8.7 & 91.3 \\
\hline Ampicillin & 8 & $>8$ & 8.7 & 91.3 \\
\hline Oxacillin & $>2$ & $>2$ & 8.7 & 91.3 \\
\hline Cephalothin & 8 & $>16$ & 56.5 & 39.1 \\
\hline Ceftriaxone & $>32$ & $>32$ & 26.1 & 60.9 \\
\hline Cefepime & $>16$ & $>16$ & 39.1 & 52.2 \\
\hline Levofloxacin & $>4$ & $>4$ & 21.7 & 73.9 \\
\hline Clindamycin & $>2$ & $>2$ & 26.1 & 69.6 \\
\hline Erythromycin & $>4$ & $>4$ & 17.4 & 73.9 \\
\hline Gentamicin & 2 & $>8$ & 56.5 & 39.1 \\
\hline Linezolid & 2 & $>4$ & 82.6 & 17.4 \\
\hline Teicoplanin & 4 & $>16$ & 82.6 & 17.4 \\
\hline Vancomycin & 2 & 4 & 95.7 & 4.3 \\
\hline Tigecycline & 0.25 & 0.5 & 100.0 & -e \\
\hline \multicolumn{5}{|l|}{ S. aureus (36) } \\
\hline Ceftobiprole & 0.5 & 2 & $100.0^{\mathrm{d}}$ & $0.0^{\mathrm{d}}$ \\
\hline Penicillin & $>8$ & $>8$ & 22.2 & 77.8 \\
\hline Ampicillin & $>8$ & $>8$ & 19.4 & 80.6 \\
\hline Cephalothin & $\leq 4$ & $>16$ & 77.8 & 16.7 \\
\hline Ceftriaxone & 4 & $>32$ & 69.4 & 13.9 \\
\hline Cefepime & 4 & $>16$ & 75.0 & 13.9 \\
\hline Clindamycin & $\leq 0.25$ & $>2$ & 77.8 & 22.2 \\
\hline Erythromycin & 1 & $>4$ & 50.0 & 44.4 \\
\hline Gentamicin & $\leq 1$ & $>8$ & 77.8 & 19.4 \\
\hline Levofloxacin & $\leq 0.5$ & $>4$ & 77.8 & 22.2 \\
\hline Linezolid & 4 & 4 & 97.2 & 2.8 \\
\hline
\end{tabular}


Table 5. In vitro activity of ceftobiprole in comparison to selected antimicrobial agents tested against the main Gram-positive pathogens collected by the INVITA-A-CEFTO Brazilian Study

\begin{tabular}{|c|c|c|c|c|}
\hline \multirow[t]{2}{*}{ Organism/ Antimicrobial agent } & \multicolumn{2}{|c|}{ MIC $(\mu \mathrm{g} / \mathrm{mL})^{\mathrm{a}}$} & \multirow[b]{2}{*}{ Susceptible $(\%)^{\mathrm{b}}$} & \multirow[b]{2}{*}{ Resistant (\%) } \\
\hline & $\mathrm{MIC}_{50}$ & $\mathrm{MIC}_{90}$ & & \\
\hline \multicolumn{5}{|l|}{ S. aureus (36) } \\
\hline Vancomycin & 1 & 2 & 100.0 & 0.0 \\
\hline Teicoplanin & $\leq 2$ & $>16$ & 100.0 & 0.0 \\
\hline Tigecycline & 0.25 & 0.5 & 97.2 & -e \\
\hline Oxacillin & 0.5 & $>2$ & 66.7 & 33.3 \\
\hline \multicolumn{5}{|l|}{ S. aureus oxacillin-resistant (12) } \\
\hline Ceftobiprole & 1 & 4 & $100.0^{\mathrm{d}}$ & $0.0^{\mathrm{d}}$ \\
\hline Penicillin & $>8$ & $>8$ & 8.3 & 91.7 \\
\hline Ampicillin & $>8$ & $>8$ & 0 & 100 \\
\hline Cephalothin & 8 & $>16$ & 50.0 & 41.7 \\
\hline Ceftriaxone & 32 & $>32$ & 8.3 & 41.7 \\
\hline Cefepime & 16 & $>16$ & 25.0 & 41.7 \\
\hline Clindamycin & $>2$ & $>2$ & 33.3 & 66.7 \\
\hline Erythromycin & $>4$ & $>4$ & 8.3 & 83.3 \\
\hline Gentamicin & 2 & $>8$ & 66.7 & 25.0 \\
\hline Levofloxacin & $>4$ & $>4$ & 33.3 & 66.7 \\
\hline Linezolid & 4 & 4 & 91.7 & 8.3 \\
\hline Vancomycin & 2 & 2 & 100.0 & 0.0 \\
\hline Teicoplanin & $\leq 2$ & $>16$ & 100.0 & 0.0 \\
\hline Tigecycline & 0.25 & 0.5 & 91.7 & -e \\
\hline \multicolumn{5}{|l|}{ S. aureus oxacillin-susceptible (24) } \\
\hline Ceftobiprole & 0.5 & 0.5 & $100.0^{\mathrm{d}}$ & $0.0^{\mathrm{d}}$ \\
\hline Penicillin & $>8$ & $>8$ & 29.2 & 70.8 \\
\hline Ampicillin & $>8$ & $>8$ & 29.2 & 70.8 \\
\hline Cephalothin & $\leq 4$ & $\leq 4$ & 91.7 & 4.2 \\
\hline Ceftriaxone & 4 & 4 & 100.0 & 0.0 \\
\hline Cefepime & 2 & 4 & 100.0 & 0.0 \\
\hline Clindamycin & $\leq 0.25$ & $\leq 0.25$ & 95.8 & 4.2 \\
\hline Erythromycin & $\leq 0.5$ & $>4$ & 70.8 & 25.0 \\
\hline Gentamicin & $\leq 1$ & $>8$ & 79.2 & 16.7 \\
\hline Levofloxacin & $\leq 0.5$ & $\leq 0.5$ & 100.0 & 0.0 \\
\hline Linezolid & 4 & 4 & 100.0 & 0.0 \\
\hline Tigecycline & 0.25 & 0.5 & 100.0 & -e \\
\hline Vancomycin & 1 & 2 & 100.0 & 0.0 \\
\hline Teicoplanin & $\leq 2$ & $\leq 2$ & 100.0 & 0.0 \\
\hline
\end{tabular}

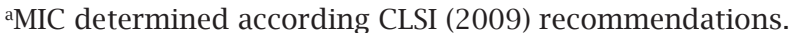

${ }^{b}$ Resistance rates calculated according CLSI M100-S21 (2011) document, except for tigecycline, for which the FDA criteria was used.

'Susceptible rates calculated considering the high level of gentamicin and streptomicin resistance.

${ }^{\mathrm{d}}$ Interpretative criteria according to Rossolini et al. ${ }^{14}$

eInterpretative criteria not established by CLSI or FDA. 
Table 6. Antimicrobial activity of ceftobiprole tested against E. faecalis, S. aureus, and CoNS isolates collected from hospitalized patients as part of the INVITA-A-CEFTO Brazilian Study

\begin{tabular}{|c|c|c|c|c|c|c|c|c|}
\hline \multirow[t]{2}{*}{ Organism (No. tested) } & \multicolumn{8}{|c|}{ Cumulative \% inhibited at MIC $(\mu \mathrm{g} / \mathrm{mL})$} \\
\hline & $\leq 0.12$ & 0.25 & 0.5 & 1 & 2 & 4 & 8 & $\geq 16$ \\
\hline E. faecalis (18) & & 33.3 & 61.1 & 88.9 & 88.9 & 88.9 & 88.9 & 100.0 \\
\hline CoNS (23) & 4.3 & 17.4 & 30.4 & 52.2 & 95.7 & 95.7 & 100.0 & \\
\hline Staphylococcus aureus (SA, 36) & & 16.7 & 72.2 & 83.3 & 94.4 & 100.0 & & \\
\hline Oxacillin-susceptible SA (24) & & 25.0 & 100.0 & & & & & \\
\hline Oxacillin-resistant SA (12) & & & 16.7 & 50.0 & 83.3 & 100.0 & & \\
\hline
\end{tabular}

observed among Gram-negative bacilli isolates collected from private hospitals were higher than those previously reported by other surveillance studies that evaluated bacterial isolates mostly collected from public/teaching hospitals. ${ }^{20}$

There has been a dramatic rise in antibiotic resistance in the hospital setting in the past decade. MRSA and carbapenem-resistant Gram-negative bacilli are of particular concern. ${ }^{6}$ There is an urgent need to expand treatment options for treating infections caused by these pathogens. The activity of ceftobiprole against the S. aureus and CoNS isolates studied was good and warrant continued evaluation of ceftobiprole as therapy for severe infections, including skin and soft tissue infections, especially in those institutions/regions where MRSA is highly prevalent.

\section{ACKNOWLEDGEMENTS}

This study was supported by an educational/research grant from Janssen-Cilag.

\section{REFERENCES}

1. Endimiani A, Perez F, Bonomo RA. Cefepime: a reappraisal in an era of increasing antimicrobial resistance. Expert Rev Anti Infect Ther 2008; 6(6):805-24.

2. Yahav D, Paul M, Fraser A, Sarid N, Leibovici L. Efficacy and safety of cefepime: a systematic review and meta-analysis. Lancet Infect Dis 2007; 7(5):338-48.

3. Gales AC, Sader HS, Ribeiro J, Zoccoli C, Barth A, Pignatari AC. Antimicrobial susceptibility of gram-positive bacteria isolated in Brazilian hospitals participating in the SENTRY Program (2005-2008). Braz J Infect Dis 2009; 13(2):90-8.

4. Noel GJ. Clinical profile of ceftobiprole, a novel beta-lactam antibiotic. Clin Microbiol Infect 2007; 13 Suppl 2:25-9.

5. Davies TA, Page MG, Shang W, Andrew T, Kania M, Bush K. Binding of ceftobiprole and comparators to the penicillin-binding proteins of Escherichia coli, , Staphylococcus aureus, and Streptococcus pneumoniae. Antimicrob Agents Chemother 2007; 51(7):2621-4.
6. Fritsche TR, Sader HS, Jones RN. Antimicrobial activity of ceftobiprole, a novel anti-methicillin-resistant Staphylococcus aureus cephalosporin, tested against contemporary pathogens: results from the SENTRY Antimicrobial Surveillance Program (2005-2006). Diagn Microbiol Infect Dis 2008; 61(1):86-95.

7. Bogdanovich T, Ednie LM, Shapiro S, Appelbaum PC. Antistaphylococcal activity of ceftobiprole, a new broad-spectrum cephalosporin. Antimicrob Agents Chemother 2005; 49(10):4210-9.

8. Bogdanovich T, Clark C, Ednie L et al. Activities of ceftobiprole, a novel broad-spectrum cephalosporin, against Haemophilus influenzae and Moraxella catarrhalis. Antimicrob Agents Chemother 2006; 50(6):2050-7.

9. Horan TC, Andrus M, Dudeck MA. CDC/NHSN surveillance definition of health care-associated infection and criteria for specific types of infections in the acute care setting. Am J Infect Control 2008; 36(5):309-32.

10. Clinical and Laboratory Standards Institute. M7-A8, Methods for dilution antimicrobial susceptibility tests for bacteria that grow aerobically; approved standard - 18th edition. Wayne, PA: CLSI, 2009.

11. Clinical and Laboratory Standards Institute. Performance standards for antimicrobial susceptibility testing: 21 st informational supplement (M100-S21), Wayne, PA: CLSI, 2011.

12. Doribax[package insert]. Raritan, NJ: Ortho-McNeil Pharmaceutical, Inc, 2007.

13. Tygacil [package insert]. Philadelphia, PA: Wyeth Pharmaceuticals Inc, 2005

14. Rossolini GM, Dryden MS, Kozlov RS et al. Comparative activity of ceftobiprole against Gram-positive and Gramnegative isolates from Europe and the Middle East: the CLASS study. J Antimicrob Chemother 2011; 66(1):151-9.

15. Zhanel GG, Voth D, Nichol K, Karlowsky JA, Noreddin AM, Hoban DJ. Pharmacodynamic activity of ceftobiprole compared with vancomycin versus methicillin-resistant Staphylococcus aureus (MRSA), vancomycin-intermediate Staphylococcus aureus (VISA) and vancomycin-resistant Staphylococcus aureus (VRSA) using an in vitro model. J Antimicrob Chemother 2009; 64(2):364-9.

16. Jones RN, Deshpande LM, Mutnick AH, Biedenbach DJ. In vitro evaluation of BAL9141, a novel parenteral cephalosporin active against oxacillin-resistant staphylococci. J Antimicrob Chemother 2002; 50(6):915-32. 
17. Queenan AM, Shang W, Kania M, Page MG, Bush K. Interactions of ceftobiprole with beta-lactamases from molecular classes A to D. Antimicrob Agents Chemother 2007; 51(9):3089-95.

18. Gales AC, Menezes LC, Silbert S, Sader HS. Dissemination in distinct Brazilian regions of an epidemic carbapenemresistant producing SPM metallo-beta-lactamase. J Antimicrob Chemother 2003; 52(4):699-702.
19. Schimith Bier KE, Luiz SO, Scheffer MC et al. Temporal evolution of carbapenem-resistant Acinetobacter baumannii in Curitiba, southern Brazil. Am J Infect Control 2010; 38(4):308-14.

20. Andrade SS, Sader HS, Barth AL et al. Antimicrobial susceptibility patterns of Gram-negative bacilli isolated in Brazilian hospitals participating in the SENTRY Program (2003-2008). Braz J Infect Dis 2008; 12(Suppl 2):3-9. 